\section{Effects of thresholding level variation in fringe binarization of multiobject joint transform correlation}

Arturo Carnicer, Ignacio Juvells, and Santiago Vallmitjana

The authors are with the Department Física Aplicada i Electrònica, Laboratori d'Optica, Universitat de Barcelona, Diagonal 647, 08028 Barcelona, Spain.

Received 2 October 1990.

0003-6935/92/081012-03\$05.00/0.

(C) 1992 Optical Society of America.

It is possible to improve the fringe binarization method of joint transform correlation by choosing a suitable threshold level.

Recently, a nonlinear image correlator was introduced ${ }^{1}$ that was based on joint transform architecture. ${ }^{2}$ This nonlinear joint transform processor uses nonlinearity at the Fourier plane by thresholding the joint power spectrum. It has been shown that the nonlinear joint transform correlator can provide a higher autocorrelation peak intensity, smaller correlation sidelobes, narrower correlation width, and better discrimination sensitivity. ${ }^{3}$

In a later study the effect of binarization of the power spectrum (fringe binarization) in a joint transform correlator for multiple targets was discussed by means of a computer simulation of a concrete example. ${ }^{4}$ The conclusions are that fringe binarization may not be usefully applied for multiple target recognition because it could produce false alarms and misses, both with noise and without noise. It can be applied only to a noise-free singletarget correlation.

In this Note, the effect of threshold variation level on binarization is discussed and the possibility of increasing the reliability of the fringe binarization method is verified.

Let $g(x, y)$ be the scene with the input targets and $h(x, y)$ be the reference object (see Fig. 1). If $f(x, y)$ is the function that represents the letter $\mathrm{E}$, we can write

$$
\begin{aligned}
& g(x, y)=f(x+2 h, y)+f(x+3 h, y)+f(x+5 h, y), \\
& h(x, y)=f(x-2 h, y) .
\end{aligned}
$$

After illumination with a coherent plane wave, the intensity distribution at the Fourier plane will be:

$$
\begin{aligned}
& I(u, v)=4 F^{2} \\
& +F^{2} \exp [i 2 \pi(-h) u]+F^{2} \exp [i 2 \pi(-2 h) u]+F^{2} \exp [i 2 \pi(-3 h) u] \\
& +F^{2} \exp [i 2 \pi(h) u]+F^{2} \exp [i 2 \pi(2 h) u]+F^{2} \exp [i 2 \pi(3 h) u] \\
& +F^{2} \exp [i 2 \pi(-4 h) u]+F^{2} \exp [i 2 \pi(-5 h) u]+F^{2} \exp [i 2 \pi(-7 h) u] \\
& +F^{2} \exp [i 2 \pi(4 h) u]+F^{2} \exp [i 2 \pi(5 h) u]+F^{2} \exp [i 2 \pi(7 h) u]
\end{aligned}
$$

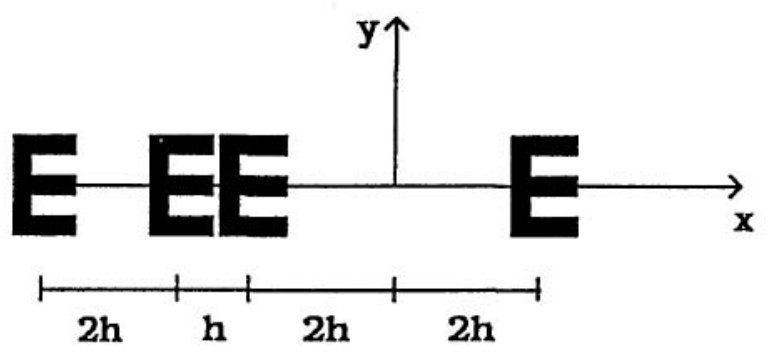

Fig. 1. Input plane geometry 1. $g(x, y)$ is the scene with three targets; $h(x, y)$ is the reference object, the letter $\mathrm{E}$.

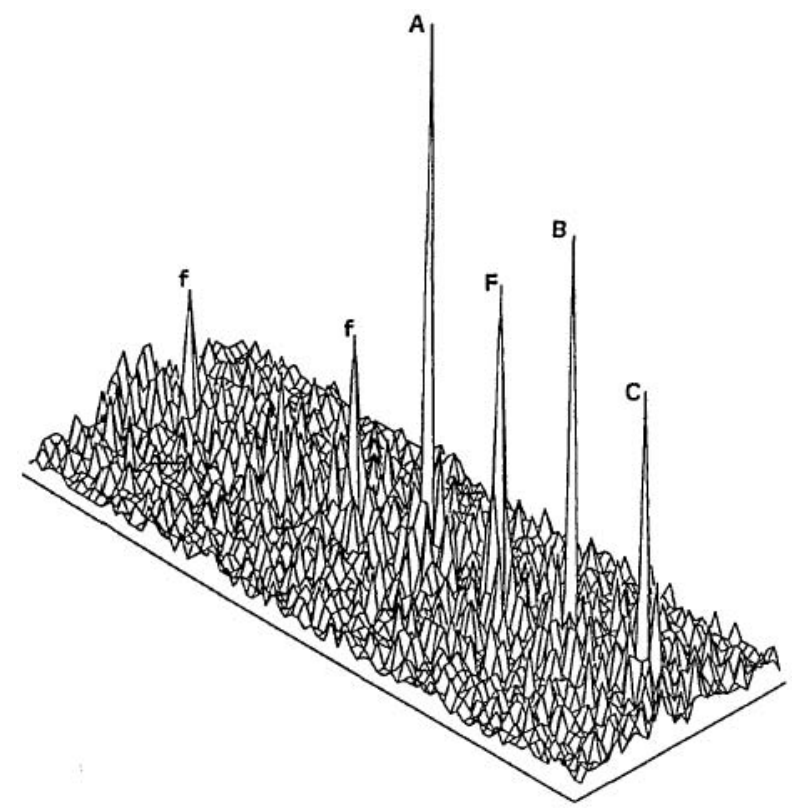

Fig. 2. Output correlation after fringe binarization with $50 \%$ of ones. $\mathrm{F}$ is the severe false alarm and $\mathrm{f}^{\prime} \mathrm{s}$ are other false correlation peaks. $\mathrm{A}, \mathrm{B}$, and $\mathrm{C}$ represent three correct correlation peaks.

where $(u, v)$ are the angular spatial frequencies and $F$ is the Fourier transform of $f(x, y)$.

The output light distribution (correlation plane amplitude), through inverse Fourier transformation, will be $U(x, y)=4(f \otimes f)$

$$
\begin{aligned}
& +(f \otimes f)^{*} \delta(x-h, y)+(f \otimes f)^{*} \delta(x-2 h, y) \\
& +(f \otimes f)^{*} \delta(x-3 h, y)+(f \otimes f)^{*} \delta(x+h, y) \\
& +(f \otimes f)^{*} \delta(x+2 h, y)+(f \otimes f)^{*} \delta(x+3 h, y) \\
& +(f \otimes f)^{*} \delta(x-4 h, y)+(f \otimes f)^{*} \delta(x-5 h, y) \\
& +(f \otimes f)^{*} \delta(x-7 h, y)+(f \otimes f)^{*} \delta(x+4 h, y) \\
& +(f \otimes f)^{*} \delta(x+5 h, y)+(f \otimes f)^{*} \delta(x-7 h, y) .
\end{aligned}
$$

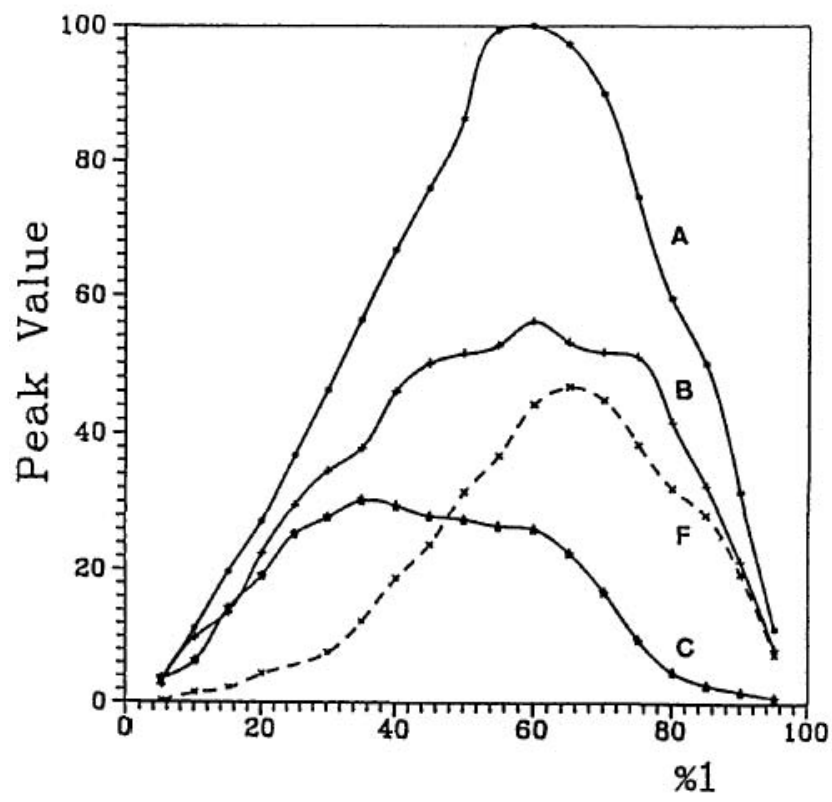

Fig. 3. Variation of intensity of correlation peaks with the percentage of ones in the binarized fringes. 


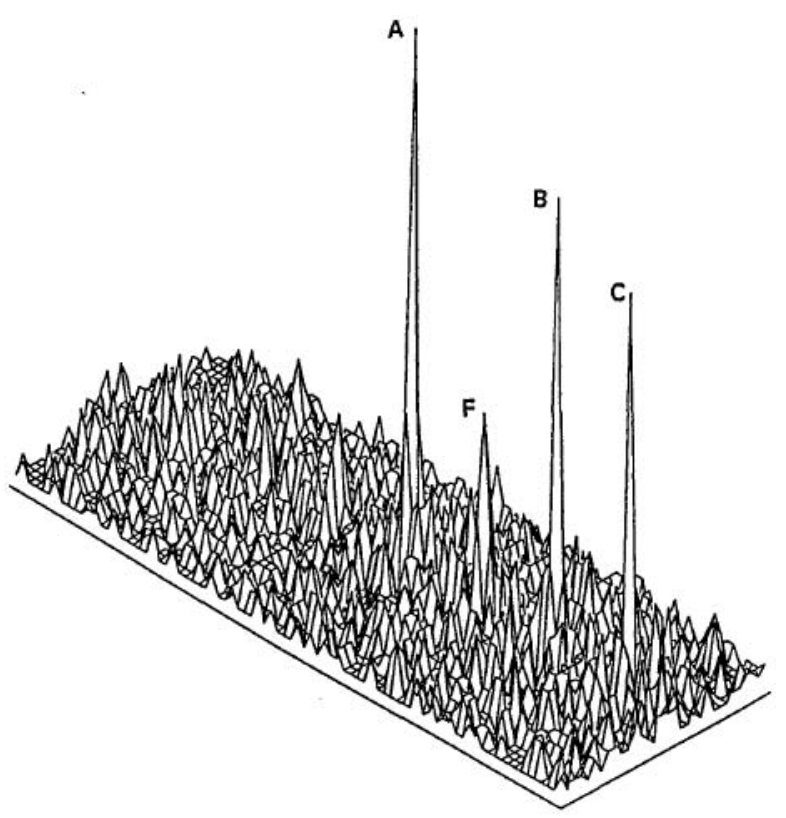

Fig. 4. Output correlation after fringe binarization with $30 \%$ of ones.

The first seven terms are the autocorrelations $g(x, y) \otimes$ $g(x, y)+f(x, y) \otimes f(x, y)$, the next three terms are the cross correlation $f(x, y) \otimes g(x, y)$, and the last three terms are the cross correlation $g(x, y) \otimes f(x, y)$.

To increase the efficiency of the method, intensity fringes are binarized according to the following equation ${ }^{1}$ :

$$
H(u, v)=\begin{aligned}
& 1, I(u, v) \geq I_{T} \\
& 0, I(u, v)<I_{T}
\end{aligned},
$$
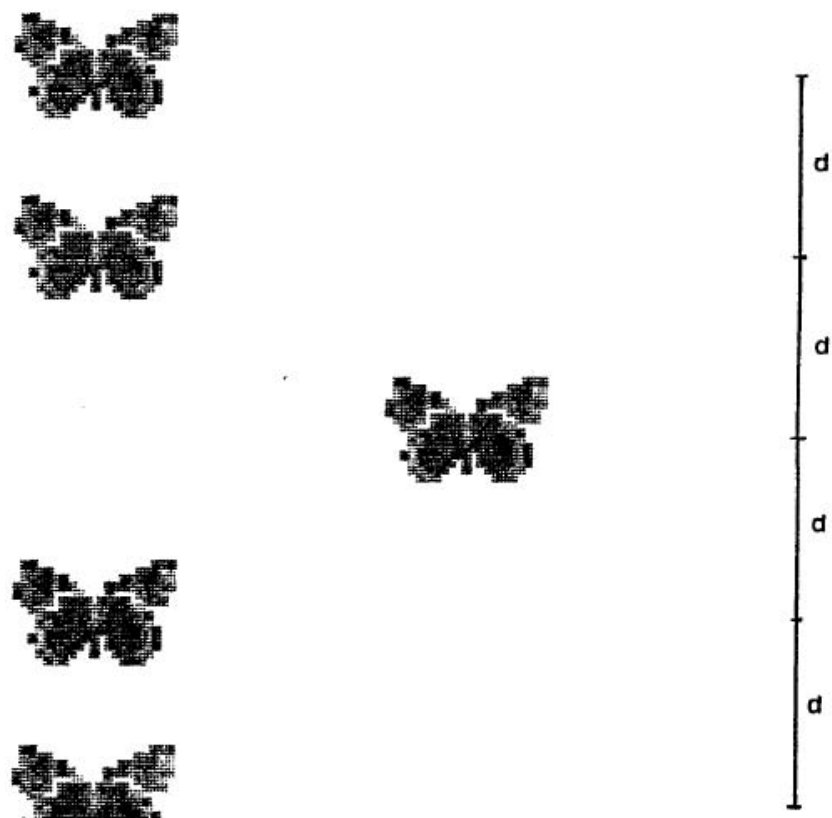

$2 d$

Fig. 5. Input plane geometry 2. $g(x, y)$ is the scene with four targets; $h(x, y)$ is the reference object, a butterfly.

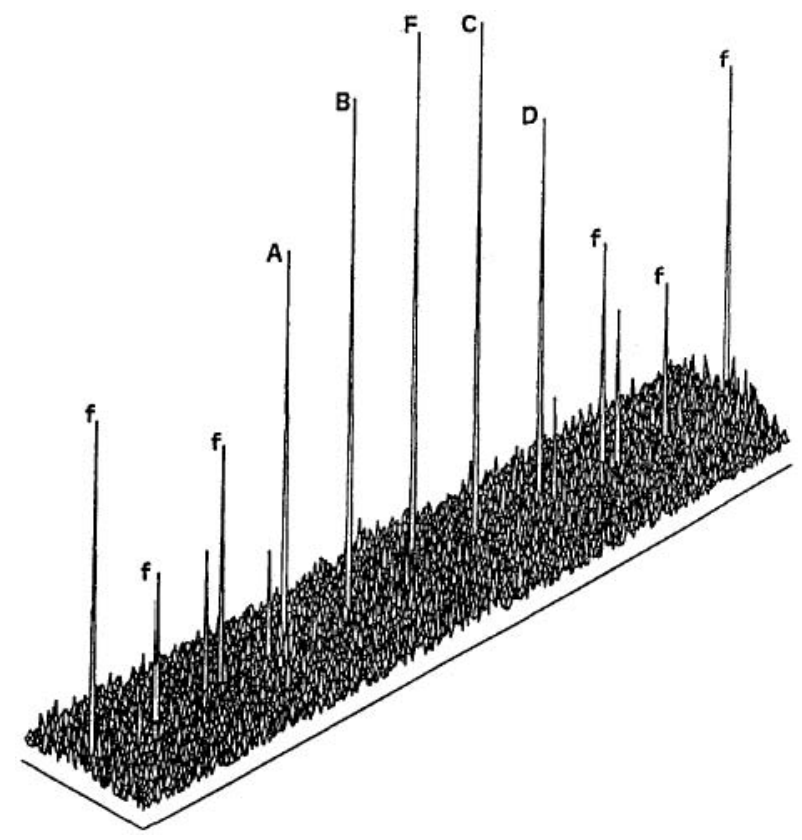

Fig. 6. Output correlation after fringe binarization with $50 \%$ of ones. $\mathrm{F}$ is the severe false alarm and $\mathrm{f}^{\prime} \mathrm{s}$ are other false correlation peaks.

where $I_{T}$ is the threshold level selected. This threshold for binarization of the intensity of the interference of the Fourier transform can be set by calculating the histogram of the pixel values of the interference intensity and then picking the median, i.e., taking the value that provides an equal number of ones and zeros.

As shown in Fig. 2, the output correlations provide false alarms in this situation because there are four correlation peaks, three correct $(A, B$, and $C)$ and one incorrect $(F)$ in

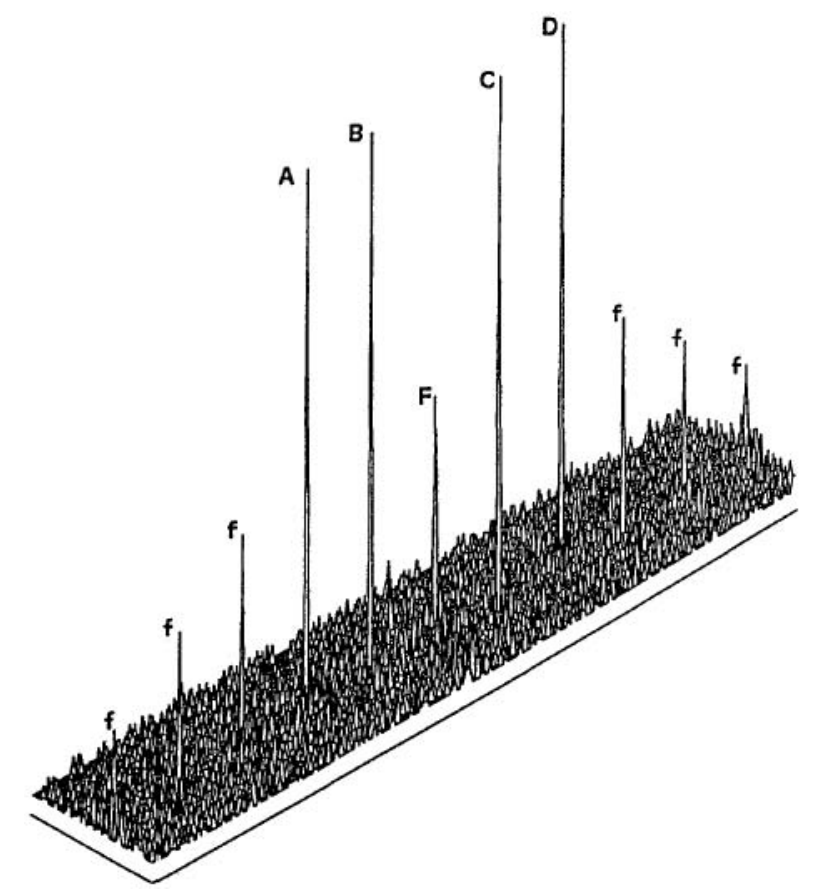

Fig. 7. Output correlation after fringe binarization with $30 \%$ of ones. 
$x= \pm 6 h{ }^{4}$ When we expand the correlation field, we also find smaller false correlation peaks $(f)$ in $x= \pm 8 h$ and $x=$ $\pm 10 h$.

However, the threshold level can take a different value from that described above. In a recent work, Javidi and Wang investigated three types of thresholding that are based on the median of the joint power spectrum in different background conditions for a single image. ${ }^{5}$

Here we have tested different threshold levels, varying the percentage of ones and zeros in the binarized power spectrum of a multiobject input scene. Figure 3 shows the variation of intensity between the four correlation peaks (A, $\mathrm{B}, \mathrm{C}$, and $\mathrm{F}$ ) and percentage of ones in the binarized Fourier-transform interference intensity, when the threshold level is changed. Peaks A, B, and C correspond to the correct identification of the letter $\mathrm{E}$, and peak $\mathrm{F}$ is a false alarm. The graphics show that when the percentage of ones is less than $45 \%$, peak $\mathrm{F}$ is the lowest, and the recognition in the output correlation plane will be correct. If the percentage of ones is $\sim 30 \%$, the discrimination between the false alarm and the correct peaks is sufficiently great. Figure 4 shows the output correlation when the threshold level is taken with $30 \%$ of ones. The $\mathrm{F}$ peak is smaller than the others, and the smaller false peaks (f peaks) have been absorbed by the correlation noise.

In order to show the applicability of variable clipping to prevent false correlation peaks, we find another scene (Fig.
5), whose correlation with a binarized joint power spectrum, taking $50 \%$ of ones, leads to a severe false alarm $(\mathrm{F})$ in $y=0 d$ and smaller false peaks (f) in $y= \pm 3 d, y= \pm 4 d$, and $y= \pm 5 d$ (Fig. 6). Figure 7 shows the output correlation when the threshold level is taken with $30 \%$ of ones.

To summarize, we have examined the effect of the variation of the threshold level in fringe binarization for a joint transform correlation. The simulated results show that, although it could introduce false alarms, the method can improve detection by choosing a suitable level of threshold, and more reliable peaks for multiple target recognition are obtained.

\section{References}

1. B. Javidi and S. F. Odeh, "Multiple object identification in bipolar joint transform correlation," Opt. Eng. 27, 295-300 (1988).

2. C. S. Weaver and J. W. Goodman, "A technique for optically convolving two functions," Appl. Opt. 5, 1248-1249 (1966).

3. B. Javidi and J. L. Horner, "Single spatial light modulator joint transform correlator," Appl. Opt. 28, 1027-1032 (1989).

4. F. T. S. Yu, F. Cheng, T. Nagata, and D. A. Gregory, "Effects of fringe binarization of multiobject joint transform correlation," Appl. Opt. 28, 2988-2990 (1989).

5. B. Javidi and J. Wang, "Binary nonlinear joint transform correlation with median and subset median thresholding," Appl. Opt. 30, 967-976 (1991). 